\title{
BIBLIOGRAPHY
}

\author{
PRINTED PRIMARY SOURCES
}

Acta pontificum Romanorum inedita. Edited by Julius Albert Georg von Pflugk-Harttung. 3 vols. Tübingen, I88I-86. Reprint. Graz, I958.

Annales Admuntenses. Edited by Wilhelm Wattenbach. MGH SS 9:569-79. Hanover, I85I.

Annales Gotwicenses a. I068-I230. Edited by Wilhelm Wattenbach. MGH SS 9:600-604. Hanover, I85I.

Annales Sancti Rudberti Salisburgenses. Edited by Wilhelm Wattenbach. MGH S S 9:758-810. Hanover, I85I.

Annalium Salisburgensium additamentum. Edited by Wilhelm Wattenbach. MGH S S 13:2364I. Hanover, I88I.

Bielsky, Wilhelm. "Die ältesten Urkunden des Kanonikatstiftes Sanct Georgen in Unterösterreich: Von III2 bis I244." Archiv für Kunde österreichischer Geschichts-Quellen 9 ( 1853): 235-304.

Chronicon Ebersheimense. Edited by Ludwig Weiland. MGH SS 23:427-53. Hanover, I874.

Chronicon Gurcense. Edited by Wilhelm Wattenbach. MGH SS 23:8-I0. Hanover, I 874.

Codex Falkensteinensis: Die Rechtsaufzeichnungen der Grafen von Falkenstein. Edited by Elisabeth Noichl. QE, n.s., 29. Munich, 1978.

Codex traditionum Augiensium. Edited by Johann Mayerhofer. In Drei bayerische Traditionsbücher aus dem XII. Jahrhundert: Festschrift zum 700 jährigen Jubiläum der Wittelsbacher Thronbesteigung, pp. 87-152. Munich, I880.

Constitutiones et acta publica imperatorum et regum. Vol. 2. Edited by Ludwig Weiland. Vol. 3 . Edited by Jakob Schwalm. MGH, Legum 4. Hanover, I896-1904/6.

Continuatio Admuntensis a. II4O-I25O, I425. Edited by Wilhelm Wattenbach. MGH SS 9:57993. Hanover, I85I.

Continuatio canonicorum Sancti Rudberti Salisburgensis. Edited by Wilhelm Wattenbach. MGH SS 9:8I9-23. Hanover, I85I.

Continuatio Claustroneoburgensis IV. a. I267-I279. Edited by Wilhelm Wattenbach. MGH SS 9:647-48. Hanover, I85I. 
Continuatio Weichardi de Polhaim a. I280-1307. Edited by Wilhelm Wattenbach. MGH SS 9:8I0-I8. Hanover, I85I.

"Epistolae Bambergenses." In Monumenta Bambergensia. Edited by Philipp Jaffé. Bibliotheca rerum Germanicarum 5. Berlin, I869.

Ficker, Julius. Forschungen zur Reichs- und Rechtsgeschichte Italiens, vol. 4. Innsbruck, I874. Reprint. Aalen, I96I.

Gerhoch of Reichersberg. Annales Reicherspergenses a. 92I-II67. Edited by Wilhelm Wattenbach. MGH SS I7:443-76. Hanover, I86I.

Hartmann von Aue. Iwein. Vol. I. Text. Edited by Georg Friedrich Benecke and Karl Lachmann and revised by Ludwig Wolff. 7th ed. Berlin, I968.

Hermann of Niederalteich. Annales et historiae Altahenses. Edited by Philipp Jaffé. MGH SS I7:35I-427. Hanover, I86I.

Historia ducum Veneticorum. Edited by Henry Simonsf eld. MGH SS 14:72-97. Hanover, I883.

Huilliard-Bréholles, Jean Louis Alphonse. Historia diplomatica Friderici Secundi; sive, Constitutiones, privilegia, mandata, instrumenta quae supersunt istius imperatoris et filiorum ejus: Accedunt epistolae paparum et documenta varia. Vol. I. Paris, I852.

Isidori Hispalensis episcopi Etymologiarum; sive, Originum libri XX. Edited by W. M. Lindsay. 2 vols. Oxford, I9II.

Magni presbyteri annales Reicherspergenses. Edited by Wilhelm Wattenbach. MGH SS 17:439534. Hanover, I86I.

Mansi, Giovanni Domenico. Sacrorum conciliorum nova et amplissima collectio. 55 vols. Florence, 1759-1962.

Monumenta Boica. 60 vols. Munich, 1763-1956.

Monumenta historica ducatus Carinthiae. Edited by August von Jaksch and Hermann Wiessner. I I vols. Klagenfurt, I 896-1972. Reprint, vols. I-4. Klagenf urt, I976-78.

Necrologia Germaniae. Vol. 2. Dioecesis Salisburgensis. Edited by Sigismund HerzbergFränkel. MGH. Berlin, 1904. Reprint. Munich, 1983. Vol. 3. Dioeceses Brixinensis, Frisingensis, Ratisbonensis. Edited by Franz Ludwig Baumann. MGH. Berlin, 1905. Reprint. Munich, 1983.

Oschinsky, Dorothea. "Südtiroler Urkunden im Britischen Museum." In Festschrift zur Feier des zweihundertjährigen Bestandes des Haus-, Hof- u. Staatsarchivs, vol. 2, edited by Leo Santifaller, pp. 529-50. Vienna, I95I.

Ottokars Österreichische Reimchronik. Edited by Joseph Seemüller. 2 vols. MGH, Deutsche Chroniken 5. Hanover, 1890-93. Reprint. Dublin, 1974.

Regesta Imperii. Vol. 5/I. Die Regesten des Kaiserreichs unter Philipp, Otto IV, Friedrich II. Heinrich (VII), Conrad IV, Heinrich Raspe, Wilhelm und Richard, II98-I272. Edited by Johann Friedrich Böhmer and Julius Ficker. Innsbruck, I88I. Vol. 6/I. Die Regesten des Kaiserreichs unter Rudolf, Adolf, Albrecht, Heinrich VII., I273-I313. Edited by Johann Friedrich Böhmer and Oswald Redlich. Innsbruck, I898.

Regesten aus dem Archive der Stadt Wien. Vol. I. Verzeichnis der Originalurkunden des städtischen Archives (1239-I4II). Edited by Karl Uhlirz. Quellen zur Geschichte der Stadt Wien 2. Vienna, 1898 .

Die Regesten der Erzbischöfe und des Domkapitels von Salzburg, I247-I343. Edited by Franz Martin. 3 vols. Salzburg, I926-34.

Regesten des Herzogtums Steiermark. Vol. I. 1308-1319. Edited by Annelies Redik. 2 pts. Quellen zur geschichtlichen Landeskunde der Steiermark 6, 7. Graz, I976-85.

Salzburger Urkundenbuch. Edited by Willibald Hauthaler and Franz Martin. 4 vols. Salzburg, I9I0-33.

Schenkungsbuch der ehemaligen gefürsteten Probstei Berchtesgaden. Edited by Karl August Muf- 
fat. Quellen und Erörterungen zur bayerischen und deutschen Geschichte I, pp. 225-364. Munich, I856.

Das "Stiftungen-Buch" des Cistercienser-Klosters Zwetl. Edited by Johann von Frast. FRA 2/3. Vienna, I85I.

Tiroler Urkundenbuch. Part I. Die Urkunden zur Geschichte des deutschen Etschlandes und des Vintschgaus. Edited by Franz Huter. 3 pts. Innsbruck, 1937-57.

Die Traditionen, Urkunden und Urbare des Stiftes Gars. Edited by Heiner Hofmann. QE, n.s., 3I. Munich, 1983.

Das Traditionsbuch des Augustiner-Chorherrenstiftes Neustift bei Brixen. Edited by Hans Wagner. FRA 2/76. Vienna, I954.

Die Traditionsbücher des Hochstifts Brixen vom Io. bis indas I4. Jahrhundert. Edited by Oswald Redlich. Acta Tirolensia: Urkundliche Quellen zur Geschichte Tirols I. Innsbruck, I886. Reprint. Aalen, 1973.

Die Traditionsnotizen des Klosters Raitenhaslach. Edited by Karlheinrich Dumrath. QE, n.s., 7. Munich, 1938. Reprint. Aalen, 1969.

Ulrich von Eschenbach. Alexander. Edited by Wendelin Toischer. Bibliothek des litterarischen Vereins in Stuttgart I83. Tübingen, I888. Reprint. New York, 1974.

Ulrich von Liechtenstein Frauendienst. Edited by Franz Viktor Spechtler. GAG 485. Göppingen, 1987.

Ulrich von Liechtenstein's "Service of Ladies." Translated by J. W. Thomas. University of North Carolina Studies in the Germanic Languages and Literatures 63. Chapel Hill, I969.

Ulrich's von Liechtenstein Frauendienst. Edited by Reinhold Bechstein, 2 vols. Deutsche Dichtungen des Mittelalters 6, 7. Leipzig, I888.

Die Urkunden der Brixner Hochstifts-Archive, 845-I295. Vol. I. Edited by Leo Santifaller. Schlern-Schriften: Veröffentlichungen zur Landeskunde von Südtirol I5. Innsbruck, 1929. Vol. 2. Edited by Leo Santifaller and Heinrich Appelt. 2 pts. Leipzig, I94I-43.

Die Urkunden des Augustiner-Chorherrenstiftes Neustift bei Brixen von II43 bis I299. Edited by Georg Johannes Kugler. FRA 2/77. Vienna, 1965.

Urkunden des Cistercienser-Stiftes Heiligenkreuz im Wiener Walde. Edited by Johann Nepomuk Weis. FRA 2/II. Vienna, I856.

Die Urkunden des Klosters Raitenhaslach, I034-1350. Edited by Edgar Krausen. 2 vols. QE, n.s., I7. Munich, 1959-60.

Die Urkunden des Rodenegg-Archivs, I288-I34O. Edited by Leo Santifaller. Schlern-Schriften: Veröffentlichungen zur Landeskunde von Südtirol 2I . Innsbruck, 1933.

Urkunden- und Regestenbuch des Herzogtums Krain. Edited by Franz Schumi. 2 vols. Ljubljana, I 882-87.

Urkundenbuch des Herzogthums Steiermark. Edited by Joseph von Zahn and Gerhard Pferschy. 4 vols. Graz, I875-1975.

Urkundenbuch des Landes ob der Enns. II vols. Vienna, I852-1956.

Urkundenbuch zur Geschichte der Babenberger in Österreich. Prepared by Oskar Frh. v. Mitis and edited by Heinrich Fichtenau and Erich Zöllner. Vol. I. Die Siegelurkunden der Babenberger bis I215. Vienna, 1950. Vol. 2. Die Siegelurkunden der Babenberger und ihrer Nachkommen von I2I 6 bis I 279. Vienna, 1955. Vol. 3. Die Siegel der Babenberger. Completed by Franz Gall. Vienna, I954. Vol. 4/I. Ergänzende Quellen, 976-II94. With the assistance of Heide Dienst. Vienna, 1968.

Vita beati Hartmanni episcopi Brixinensis (II4O-II64). Edited by Anselm Sparber. SchlernSchriften 46. Innsbruck, 1940.

Vita Chunradi archiepiscopi Salisburgensis. Edited by Wilhelm Wattenbach. MGH SS II :62-77. Hanover, I854. 
Vita Gebehardi archiepiscopi Salisburgensis. Edited by Wilhelm Wattenbach. MGH SS II :2528. Hanover, 1854 .

Vita Gebehardi et successorum eius. Edited by Wilhelm Wattenbach. MGH SS II :33-49. Hanover, I 854 .

\section{SECONDARY LITERATURE}

Ackermann, Horst. "Die Iwein-Fresken auf Schloss Rodenegg." Der Schlern 57 (I983): 39I421 .

Adelige Sachkultur des Spätmittelalters: Internationaler Kongress Krems an der Donau 22. bis 25. September 1980. Veröffentlichungen des Instituts für mittelalterliche Realienkunde Österreichs 5. Österreichische Akademie der Wissenschaften: Philosophisch-historische Klasse 400. Vienna, 1982.

Das älteste Kloster im deutschen Sprachraum: St. Peter in Salzburg: 3. Landesausstellung 15. Mai-26. Oktober 1982: Schätze europäischer Kunst und Kultur. Salzburg, 1982.

Althoff, Gerd. Adels- und Königsfamilien im Spiegel ihrer Memorialüberlieferung: Studien zum Totengedenken der Billunger und Ottonen. Münstersche Mittelalter-Schriften 47. Munich, 1984 .

— . "Königsherrschaft und Konfliktbewältigung im Io. und I I. Jahrhundert." Frühmittelalterliche Studien: Jahrbuch des Instituts für Frühmittelalterforschung der Universität Münster 23 (I989): 265-90.

_. "Nunc fiant Christi milites, qui dudum extiterunt raptores: Zur Entstehung von Rittertum und Ritterethos." Saeculum: Jahrbuch für Universalgeschichte 32 (I98I): 317-33.

- Verwandte, Freunde und Getreue: Zum politischen Stellenwert der Gruppenbindungen im früheren Mittelalter. Darmstadt, 1990.

Arnold, Benjamin. Count and Bishop in Medieval Germany: A Study of Regional Power, IIOO1350. Philadelphia, I99I .

_ "German Bishops and Their Military Retinues in the Medieval Empire." German History 7 (I989): I6I-83.

- German Knighthood, IO50-1300. Oxford, 1985.

_. Princes and Territories in Medieval Germany. Cambridge, I99I .

Arnold, Klaus. "Admont und die monastische Reform des I2. Jahrhunderts." Z(S)RG, KA 89 (1972): 350-69.

Barker, Juliet, and Maurice Keen. "The Medieval English Kings and the Tournament." In Fleckenstein, Das ritterliche Turnier, pp. 21 2-28.

Barnes, R. H. "Marriage, Exchange and the Meaning of Corporations in Eastern Indonesia." In Comaroff, Meaning of Marriage Payments, pp. 93-124.

Baumgartner, Egon. "Beiträge zur Geldgeschichte der Friesacher Pfennige." Carinthia I. I 50/ I (1960): 84-117.

Benediktinerabtei Michaelbeuern: Eine Dokumentation anlässlich der Eröffnung und Weihe der neu adaptierten Räume für Internat, Schule und Bildungsarbeit. Michaelbeuern, 1985.

Bilowitzky, Harald. "Die Heiratsgaben in der Steiermark während des späten Mittelalters unter stände- und wirtschaftsgeschichtlichem Aspekt.” Ph.D. diss., University of Graz, 1977.

Birlauf-Bonnet, Anne Marie. "Überlegungen zur Brixner Malerei in den ersten Jahrzehnten des I 3. Jahrhunderts." Wiener Jahrbuch für Kunstgeschichte 37 (1984): 23-39, I87-98.

Bitschnau, Martin. Burg und Adel in Tirol zwischen I050 und 1300: Grundlagen zu ihrer Erforschung. Mitteilungen der Kommission für Burgenforschung und Mittelalter-Archäologie, 
Sonderband I. Österreichische Akademie der Wissenschaften, Philosophisch-historische Klasse 403. Vienna, I983.

Bloch, Marc. Feudal Society. Translated by L. A. Manyon. 2 vols. Chicago, 1964.

Bonnet, Anne-Marie. Rodenegg und Schmalkalden: Untersuchungen zur Illustration einer ritterlich-höfischen Erzählung und zur Entstehung profaner Epenillustration in den ersten Jahrzehnten des 13. Jahrhunderts. tuduv-Studien [sic]: Reihe Kunstgeschichte 22. Munich, 1986.

Borst, Arno. "Das Rittertum im Hochmittelalter: Idee und Wirklichkeit." Saeculum: Jahrbuch für Universalgeschichte Io (1959): 213-3I. Reprinted in Borst, Das Rittertum im Mittelalter, pp. 212-46. WF 349. Darmstadt, 1976.

Bosl, Karl. Europa im Aufbruch: Herrschaft, Gesellschaft, Kultur vom Io. bis zum I4. Jahrhundert. Munich, 1980.

- Die Grundlagen der modernen Gesellschaft im Mittelalter: Eine deutsche Gesellschaftsgeschichte des Mittelalters. 2 vols. Monographien zur Geschichte des Mittelalters 4. Stuttgart, 1972.

Die Reichsministerialität der Salier und Staufer: Ein Beitrag zur Geschichte des hochmittelalterlichen deutschen Volkes, Staates und Reiches. MGH Schriften I0. Stuttgart, 1950-5I.

" "Vorstufen der deutschen Königsdienstmannschaft (Begriffsgeschichtlich-prosopographische Studien zur frühmittelalterlichen Sozial- und Verfassungsgeschichte)." In Frühformen der Gesellschaft im mittelalterlichen Europa: Ausgewählte Beiträge zu einer Strukturanalyse der mittelalterlichen Welt, pp. 228-76. Munich, 1964.

Bouchard, Constance Brittain. "Consanguinity and Noble Marriages in the Tenth and Eleventh Centuries." Speculum 56 (1981): 268-87.

- Holy Entrepreneurs: Cistercians, Knights, and Economic Exchange in Twelfth-Century Burgundy. Ithaca, N.Y., I99I .

- Spirituality and Administration: The Role of the Bishop in Twelf th-Century Auxerre. Speculum Anniversary Monographs 5. Cambridge, Mass., 1979.

- Sword, Miter, and Cloister: Nobility and the Church in Burgundy, 980-1198. Ithaca, N.Y., 1987.

Bracher, Karl. "Lassnitz-Sulm: Zur mittelalterlichen Geschichte der Zwischenflusslandschaft." ZsHVStm 59 (1968): 135-69.

Brauneder, Wilhelm Die Entwicklung des Ehegüterrechts in Österreich: Ein Beitrag zur Dogmengeschichte und Rechtstatsachenforschung des Spätmittelalters und der Neuzeit. Salzburg, 1973.

Breinbauer, Josef. Otto von Lonsdorf: Bischof von Passau, I254-1265. Passauer historische Forschungen 6. Cologne, 1992.

Brooke, Christopher N. L. The Medieval Idea of Marriage. Oxford, 1989.

Brown, Elizabeth A. R. "The Tyranny of a Construct: Feudalism and Historians of Medieval Europe.” AHR 79 (1974): 1063-88.

Brugger, Walter. "Die Kuchler: Ein Salzburger Ministerialengeschlecht vom 12.-15. Jahrhundert." Das Salzfass, n.s., 2 (1967-68): I-33.

Brugger, Walter, Heinz Dopsch, and Peter F. Kramml, eds. Geschichte von Berchtesgaden: Stift-Markt-Land. Vol. I. Zwischen Salzburg und Bayern (bis 1594). Berchtesgaden, I99I .

Brundage, James A. Law, Sex, and Christian Society in Medieval Europe. Chicago, 1987.

Brunner, Otto. Land and Lordship: Structures of Governance in Medieval Austria. Translated by Howard Kaminsky and James Van Horn Melton. Philadelphia, 1992.

Bumke, Joachim. The Concept of Knighthood in the Middle Ages. Translated by W. T. H. Jackson and Erika Jackson. AMS Studies in the Middle Ages 2. New York, 1982.

. Courtly Culture: Literature and Society in the High Middle Ages. Translated by Thomas Dunlap. Berkeley, Calif., I99I . 
Comaroff, John L., ed. The Meaning of Marriage Payments. Studies in Anthropology 7. London, I980.

Cormeau, Christoph. "Hartmann von Aue." In Die deutsche Literatur des Mittelalters: Verfasserlexikon, vol. 3, edited by Christine Stöllinger, pp. 500-520. New York, I98I .

Crusius, Irene. "Bischof Konrad II. von Hildesheim: Wahl und Herkunft." In Institutionen, Kultur und Gesellschaft im Mittelalter: Festschrift für Josef Fleckenstein zu seinem 65. Geburtstag, edited by Lutz Fenske, Werner Rösener, and Thomas Zotz, pp. 43I-68. Sigmaringen, I 984.

Curschmann, Michael. "Herrand von Wildonie (Wildon)." In Die deutsche Literatur des Mittelalters: Verfasserlexikon, vol. 3, edited by Christine Stöllinger, I I44-47. New York, I 98 I .

Diemer, Peter, and Dorothea Diemer. “'Qui pingit florem non pingit odorem': Die Illustrationen der Carmina Burana (Clm 4660)." Jahrbuch des Zentralinstituts für Kunstgeschichte 3 (I987): 43-75.

Dollinger, Philippe. L'évolution des classes rurales en Bavière depuis la fin de l'époque carolingienne jusqu' au milieu du XIIIe siècle. Publications de la Faculté des lettres de l'Université de Strasbourg II 2. Paris, 1949.

Dopsch, Heinz. "Der bayerische Adel und die Besetzung des Erzbistums Salzburg im Io. und I I. Jahrhundert." MGSL I I0-I I (I970-7I): I25-5I.

_ . "Burgenbau und Burgenpolitik des Erzstiftes Salzburg im Mittelalter." In Die Burgen im deutschen Sprachraum: Ihre rechts- und verfassungsgeschichtliche Bedeutung, edited by Hans Patze, 2:387-417. 2 vols. VF I9. Sigmaringen, 1976.

_ . "Der Dichter Ulrich von Liechtenstein und die Herkunft seiner Familie." In Festschrift

Friedrich Hausmann, edited by Herwig Ebner, pp. 93-II 8. Graz, 1977.

—_ . "Die Frühzeit Salzburgs." In Drabek, Österreich im Hochmittelalter, pp. I 55-93.

. Geschichte Salzburgs: Stadt und Land. Vol. I. Vorgeschichte, Altertum, Mittelalter. 3 pts. Salzburg, I98I-84.

— . "Die Grafen von Lebenau." Das Salzfass, n.s., 4 (1970): 33-59.

"Die Gründer kamen vom Rhein: Die Spanheimer als Stifter von St. Paul." In Schatzhaus Kärntens: Landesausstellung St. Paul I99I, 900 Jahre Benediktinerstift, ed. Günther Hödl, vol. 2, Beiträge, pp. 43-67. Klagenfurt, I991.

"Die Ministerialität des Herzogtums Steiermark zur Zeit der Georgenberger Handf este: Ihre rechtliche, gesellschaftliche und politische Stellung." In 8oo Jahre Georgenberger Handfeste: Lebensformen im Mittelalter: Ausstellung im Museum Lauriacum Enns 15. Mai bis 26. Oktober 1986. Mitteilungen des Museumsvereins Lauriacum-Enns, n.s., 24 (1986): 29-44.

. "Ministerialität und Herrenstand in der Steiermark und in Salzburg." ZsHVStm 62 (197I): 3-3I.

—_. "Přemysl Ottokar II. und das Erzstift Salzburg." JbfLKNÖ 44-45 (1 978-79): 470-508.

"Probleme ständischer Wandlung beim Adel Österreichs, der Steiermark und Salzburg vornehmlich im I3. Jahrhundert." In Herrschaft und Stand: Untersuchungen zur Sozialgeschichte im 13. Jahrhundert, edited by Josef Fleckenstein, pp. 207-53. VMPIG 5I. Göttingen, 1977 .

. "Steinbrünning und seine Herrschaftsgeschichte." Das Salzfass, n.s., 7 (1973): 2-3I .

"Die Stifterfamilie des Klosters Gurk und ihre Verwandtschaft." Carinthia I. I6I/I (197I): 95-123.

—. "Von der Existenzkrise zur Landesbildung-Berchtesgaden im Hochmittelalter." In Brugger, Dopsch, and Kramml, Geschichte von Berchtesgaden, I:265-386.

— . "Von der Slawenmission zur Grundherrschaft: Zur Rolle des Erzbistums Salzburg und der Salzburger Klöster in Niederösterreich." In Die bayerischen Hochstifte und Klöster in der Geschichte Niederösterreichs, edited by Helmuth Feigl, pp. I-30. Studien und Forschungen aus dem niederösterreichischen Institut für Landeskunde II . Vienna, I989.

—_ . "Zur Entstehung der erzbischöflichen Herrschaft und der Landeshoheit im Gerichtsbezirk 
Radstadt." In Die alte Stadt im Gebirge: 700 Jahre Radstadt, edited by Friederike Zaisberger and Fritz Koller, pp. 29-45. Radstadt, 1989.

—. "Zur Geschichte der Burg Kalham." MGSL I 12-13 (1972-73): 265-76.

Dopsch, Heinz, and John B. Freed. "Henndorf im Mittelalter und an der Wende zur Neuzeit." In Henndorf am Wallersee: Kultur und Geschichte einer Salzburger Gemeinde, edited by Alfred Stefan Weiss, Karl Ehrenfellner, and Sabine Falk, pp. 42-97, 526-27. Henndorf, 1992.

Drabek, Anna M., ed. Österreich im Hochmittelalter (907 bis I246). Veröffentlichungen der Kommission für die Geschichte Österreichs I7. Vienna, I99I.

Dubled, Henri. "Noblesse et féodalité en Alsace du XIe au XIIIe siècle." Tijdschrift voor Rechtsgeschiedenis (Revue d' histoire du droit) 28 (1960): 129-80.

Duby, Georges. The Chivalrous Society. Translated by Cynthia Postan. Berkeley and Los Angeles, 1977.

- The Knight, the Lady, and the Priest: The Making of ModernMarriage in Medieval France. Translated by Barbara Bray. New York, 1983.

- "Lineage, Nobility and Knighthood: The Mâconnais in the Twelfth Century-A Revision." In Duby, Chivalrous Society, pp. 59-80.

- Medieval Marriage: Two Models from Twelfth-Century France. Translated by Elborg Forster. Johns Hopkins Symposia in Comparative History I I. Baltimore, 1978.

- Rural Economy and Country Life in the Medieval West. Translated by Cynthia Postan. Columbia, S.C., I968.

— . "Youth in Aristocratic Society: Northwestern France in the Twelfth Century," in Duby, Chivalrous Society, pp. I1 2-22.

Duggan, Lawrence C. Bishop and Chapter: The Governance of the Bishopric of Speyer to 1552. New Brunswick, N.J., I978.

Ebenbauer, Alfred, Fritz Peter Knapp, and Ingrid Strasser, eds. Österreichische Literatur zur Zeit der Babenberger: Vorträge der Lilienfelder Tagung 1976. Wiener Arbeiten zur germanischen Altertumskunde und Philologie Io. Vienna, 1977.

Ebner, Herwig. Das Freie Eigen: Ein Beitrag zur Verfassungsgeschichte des Mittelalters. Aus Forschung und Kunst 2. Klagenfurt, 1969.

- "Die politische und verfassungsrechtliche Stellung der Traungauer in der ehemaligen Untersteiermark." In Pferschy, Das Werden der Steiermark, pp. 277-307.

Englbrecht, Jolanda. Drei Rosenfür Bayern: Die Grafen zu Toerring von den Anfängen bis heute. Pfaffenhofen, 1985 .

Erben, Wilhelm. Mühldorfer Ritterweihen der Jahre 1319 und 1322. Veröffentlichungen des historischen Seminars der Universität Graz I 2. Graz, 1932.

Fajkmajer, Karl. "Die Ministerialen des Hochstiftes Brixen im Mittelalter." Zeitschrift des Ferdinandeums für Tirol und Voralberg, 3d ser., 52 (1908): 95-192.

Feldbauer, Peter. Der Herrenstand in Oberösterreich: Ursprünge, Anfänge, Frühformen. Sozialund wirtschaftshistorische Studien. Munich, 1972.

— Herrschaftsstruktur und Ständebildung: Beiträge zur Typologie der österreischischen Länder aus ihren mittelalterlichen Grundlagen. Vol. I. Herren und Ritter. Sozial- und wirtschaftshistorische Studien. Munich, 1973.

— . "Rangprobleme und Konnubium österreichischer Landherrenfamilien: Zur sozialen Mobilität einer spätmittelalterlichen Führungsgruppe." ZsbLG 35 (1972): 57I-90.

Fenske, Lutz. "Adel und Rittertum im Spiegel früher heraldischer Formen und deren Entwicklung." In Fleckenstein, Das ritterliche Turnier, pp. 75-160.

Festschrift St. Peter zu Salzburg, 582-1982. Studien und Mitteilungen zur Geschichte des Benediktiner-Ordens und seiner Zweige 93. Salzburg, 1982.

Fichtenau, Heinrich. Das Urkundenwesen in Österreich vom 8. bis zum frühen I3. Jahrhundert. MIÖG, suppl. vol. 23. Vienna, I97I. 
Ficker, Julius. Vom Heerschilde: Ein Beitrag zur deutschen Reichs- und Rechtsgeschichte. Innsbruck, I862.

Fleckenstein, Josef, ed. Das ritterliche Turnier im Mittelalter: Beiträge zu einer vergleichenden Formen- und Verhaltensgeschichte des Rittertums. VMPIG 80. Göttingen, 1985.

Flohrschütz, Günther. "Die Freisinger Dienstmannen im IO. und I I. Jahrhundert." Beiträge zur altbayerischen Kirchengeschichte 25 (1967): 9-79.

— . "Die Freisinger Dienstmannen im I2. Jahrhundert." Oberbayerisches Archiv 97 (1973): 32-339.

—. "Die Vögte von Mödling und ihr Gefolge." ZsbLG 38 (1975): 3-I43.

Fox, Robin. Kinship and Marriage: An Anthropological Perspective. Cambridge, 1983.

Fräss-Ehrfeld, Claudia. Geschichte Kärntens. Vol. I. Das Mittelalter. Klagenfurt, 1984.

Freed, John B. The Counts of Falkenstein: Noble Self-Consciousness in Twelfth-Century Germany. Transactions of the American Philosophical Society 74/6. Philadelphia, 1984.

_ . "The Crisis of the Salzburg Ministerialage, 1270-1343." Studies in Medieval and Renaissance History, n.s., I I (1989): II I-62.

. "Devotion to St James and Family Identity: The Thurns of Salzburg." JMH I3 (1987): 207-22.

_ . "Diemut von Högl: Eine Salzburger Erbtochter und die erzbischöfliche Ministerialität im Hochmittelalter." MGSL I 20-2I ( I980-8I ): 58I-657.

— . "Die Dienstmannschaft von St. Peter." In Festschrift St. Peter zu Salzburg, 582-1982, pp. 56-78. Studien und Mitteilungen zur Geschichte des Benediktiner-Ordens und seiner Zweige 93. Salzburg, I982.

— . "The Formation of the Salzburg Ministerialage in the Tenth and Eleventh Centuries: An Example of Upward Social Mobility in the Early Middle Ages." Viator 9 (1978): 67-102.

— . "German Source Collections: The Archdiocese of Salzburg as a Case Study." In Medieval Women and the Sources of Medieval History, edited by Joel T. Rosenthal, pp. 80-I2I. Athens, Ga., 1990 .

_ "The Ministerials and the Church in Twelfth-Century Salzburg." Medieval Prosopography 3/2 (1982): I-20.

. "Nobles, Ministerials, and Knights in the Archdiocese of Salzburg." Speculum 62 ( 1987): 575-6II

. "The Origins of the European Nobility: The Problem of the Ministerials." Viator 7 (I976): 2 II -4 I.

_ . "The Prosopography of Ecclesiastical Elites: Some Methodological Considerations from Salzburg." Medieval Prosopography 9/I (1988): 33-59.

—. "Reflections on the Medieval German Nobility." AHR 9I (1986): 553-75.

— . "Rudolf of Habsburg, the Dominicans, and the Pettaus." In Zur Sozial- und Begriffsgeschichte des Mittelalters, pp. 73-I03. Tel Aviver Jahrbuch für deutsche Geschichte 22. Gerlingen, 1993 .

Freed, John B., and Heinz Dopsch. "Die Herren von Saalfelden." In Chronik Saalfelden, edited by Alois Eder, I:9I-96. 2 vols. Innsbruck, 1992.

Fried, Johannes. "Die Rezeption bologneser Wissenschaft in Deutschland während des I 2. Jahrhunderts." Viator 2 I (I990): 103-45.

Fritz, Gerhard. Die Geschichte der Grafschaft Löwenstein und der Grafen Löwenstein-Habsburg vom späten 13. bis zurMitte des I5. Jahrhunderts. Forschungen aus Württembergisch Franken 29. Sigmaringen, 1986.

Geiss, Ernest. "Geschichte des regulirten Augustiner-Chorherren-Stiftes Högelwerd.” Beyträge zur Geschichte, Topographie und Statistik des Erzbisthums München und Freysing 4 (I852): 3I9-553.

Goody, Jack. The Development of the Family and Marriage in Europe. Cambridge, 1983. 
Hauck, Karl. "Heldendichtung und Heldensage als Geschichtsbewusstsein." In Alteuropa und die moderne Gesellschaft: Festschrift für Otto Brunner, pp. II8-69. Göttingen, I963.

Hausner, Renate. "Ulrichs von Liechtenstein 'Frauendienst': Eine steirisch-österreichische Adaption des Artusromans: Überlegungen zur Struktur." In Festschrift für Adalbert Schmidt zum 70. Geburtstag, edited by Gerlinde Weiss, pp. I2I-92. Stuttgarter Arbeiten zur Germanistik 4. Stuttgart, 1976.

_ . "Ulrichs von Liechtenstein 'Frauendienst' - eine steirisch-österreichische Adaption des Artusromans." In Ebenbauer, Knapp, and Strasser, Österreichische Literatur zur Zeit der Babenberger, pp. 50-67.

Hemma von Gurk: Katalog: Ausstellung auf Schloss Strassburg/Kärnten: I4. Mai bis 26. Oktober 1988. Klagenfurt, 1988.

Herlihy, David. "The Making of the Medieval Family: Symmetry, Structure, and Sentiment." Journal of Family History 8 (1983): I $16-30$.

- Medieval Households. Cambridge, Mass., 1985.

Higounet, Charles. Les Allemands en Europe centrale et orientale au Moyen Age. Paris, 1989.

Höck, Josef. Geschichte der Propstei Wieting im Görtschitztal, Kärnten (1I47-I848). Salzburg, I979.

Höfler, Otto. "Ulrichs von Liechtenstein Venusfahrt und Artusfahrt." In Studien zur deutschen Philologie des Mittelalters: Friedrich Panzer zum 8o. Geburtstag am 4. September 1950 dargebracht, edited by Richard Kienast, pp. 13I-52. Heidelberg, 1950.

Huffman, Joseph P. "Prosopography and the Anglo-Imperial Connection: A Cologne Ministerialis Family and Its English Relations." Medieval Prosopography II/2 (I990): 53-I34.

Jackman, Donald C. The Konradiner: A Study in Genealogical Methodology. Ius Commune: Veröffentlichungen des Max-Planck-Instituts für europäische Rechtsgeschichte, suppl. vol. 47. Frankfurt, 1990.

Jackson, William Henry. "Das Turnier in der deutschen Dichtung des Mittelalters." In Fleckenstein, Das ritterliche Turnier, pp. 257-95.

Jaksch, August. Geschichte Kärntens bis 1335. 2 vols. Klagenfurt, 1928-29.

Johanek, Peter. "Zur rechtlichen Funktion von Traditionsnotiz, Traditionsbuch und früher Siegelurkunde." In Recht und Schrift im Mittelalter, edited by Peter Classen, pp. I3I-62. VF 23. Sigmaringen, 1977.

Johnson, Penelope D. Equal in Monastic Profession: Religious Women in Medieval France. Chicago, I991.

Kaiser, Gert. Textauslegung und gesellschaftliche Selbstdeutung: Die Artusromane Hartmanns von Aue. $2 \mathrm{~d}$ ed. Wiesbaden, 1978.

Kaminsky, Howard. "Estate, Nobility, and the Exhibition of Estate in the Later Middle Ages." Speculum 68 (1993): 684-709.

Kirmeier, Josef, and Evamaria Brockhoff, eds. Herzöge und Heilige: Das Geschlecht der Andechs-Meranier im europäischen Hochmittelalter: Katalog zur Landesausstellung im Kloster Andechs 13. Juli-24. Oktober 1993. Veröffentlichungen zur bayerischen Geschichte und Kultur 24/93. Munich, I993.

Klassen, John. "Household Composition in Medieval Bohemia." JMH I6 (1990): 55-75.

Klebel, Ernst. Der Lungau: Historisch-politische Untersuchung. Salzburg, I960.

Klein, Herbert. "Die bäuerlichen Eigenleute des Erzstifts Salzburg im späteren Mittelalter." In Beiträge zur Siedlungs-, Verfassungs- und Wirtschaftsgeschichte von Salzburg: Gesammelte Aufsätze von Herbert Klein: Festschrift zum 65. Geburtstag von Herbert Klein, pp. 137-251. MGSL, suppl. vol. 5. Salzburg, I965.

_. "Salzburg, ein unvollendeter Pass-Staat." In Die Alpen in der europäischen Geschichte des Mittelalters: Reichenau-Vorträge, 196I-1962, pp. 275-9I. VF Io. Sigmaringen, 1965. 
"Salzburg und seine Landstände von den Anfängen bis I86I." In Beiträge zur Siedlungs-, Verfassungs- und Wirtschaftsgeschichte von Salzburg, pp. I 5-36.

_. "Zur älteren Geschichte der Salinen Hallein und Reichenhall." In Beiträge zur Siedlungs-, Verfassungs- und Wirtschaftsgeschichte von Salzburg, pp. 385-409.

Klos-Buzek, Friederike. "Zur Frage der 'vita canonica' im Brixner Domkapitel während des Hochmittelalters." MIÖG 67 (I959): IOI-I6.

Koch, Bernhard. "Der Salzburger Pfennig: Münz- und Geldgeschichte Salzburgs im Mittelalter." Numismatische Zeitschrift 75 (1953): 36-73.

Koch-Sternfeld, Joseph Ernst Ritter von. Salzburg und Berchtesgaden in historisch-statistischgeographisch- und staatsökonomischen Beyträgen. 2 vols. Salzburg, I8Io.

Köhler, Erich. Ideal und Wirklichkeit in der höfischen Epik: Studien zur Form der frühen Artusund Graldichtung. Beihefte zur Zeitschrift für romanische Philologie 97. Tübingen, 1956.

Koller, Fritz. "Hallein im frühen und hohen Mittelalter." MGSL II6 (I976): I-II6.

Koller, Heinrich. "König Arnolfs grosses Privileg für Salzburg." MGSL Io 9 ( I969): 65-75.

Koller-Neumann, Irmtraud. Die Lehen des Bistums Bamberg in Kärnten bis I4Oo. Das Kärntner Landesarchiv 7. Klagenfurt, 1982.

Kramml, Peter F. "Propstei und Land Berchtesgaden im Spätmittelalter: Das Ringen mit Salzburg um politische, wirtschaftliche und kirchliche Selbständigkeit." In Brugger, Dopsch, and Kramml, Geschichte von Berchtesgaden I:387-542.

Krausen, Edgar. Das Erzbistum Salzburg. Vol. I . Die Zisterzienserabtei Raitenhaslach. Germania Sacra, n.s., I I, Die Bistümer der Kirchenprovinz Salzburg. New York, 1977.

Die Kuenringer: Das Werden des Landes Niederösterreich: Niederösterreichische Landesausstellung Stift Zwettl: I6. Mai-26. Okt. I98I. Katalog des niederösterreichischen Landesmuseum, n.s., I Io. Vienna, I98I.

Lackner, Christian. "Zur Geschichte der Grafen von Ortenburg in Kärnten und Krain." Carinthia I. I8I (I99I): I8I-200.

Landau, Peter. "Hadrians IV. Dekretale 'Dignum est' (X.4.9.I) und die Eheschliessung Unfreier in der Diskussion von Kanonisten und Theologen des I2. und I3. Jahrhunderts." Studia Gratiana I 2 ( 1967): 5I I-53.

Lang, Alois, and Gustaf Adolf von Metnitz. Die Salzburger Lehen in Kärnten bis 1520. FRA 2/79. Vienna, I97I.

Lau, Friedrich. "Das kölner Patriziat bis zum Jahre 1325." Mitteilungen aus dem Stadtarchiv von Köln 9 ( I894): 65-89, 358-8I; I0 (I895): I03-58.

Lechner, Karl. Die Babenberger: Markgrafen und Herzoge von Österreich, 976-I246. Veröffentlichungen des Instituts für österreichische Geschichtsforschung 23. Vienna, 1976. Reprint. Darmstadt, 1985.

—_ . "Zur älteren Geschichte von Zöbing und seines Herrengeschlechtes.” In 850 Jahre Zöbing am Kamp (70o Jahre Pfarre), pp. I3-3I. Zöbing am Kamp, 1958.

Le Roy Ladurie, Emmanuel. Times of Feast, Times of Famine: A History of Climate since the Year IOoo. Translated by Barbara Bray. New York, I97I.

Lévi-Strauss, Claude. "Structural Analysis in Linguistics and in Anthropology." In Structural Anthropology, translated by Claire Jacobson and Brooke Grundf est Schoepf, pp. 29-53. Garden City, N.Y., 1967.

Leyser, Karl J. Rule and Conflict in an Early Medieval Society: Ottonian Saxony. Bloomington, Ind., 1979.

Lhotsky, Alphons. Quellenkunde zur mittelalterlichen Geschichte Österreichs. MIÖG, suppl. vol. I9. Graz, 1963 .

Liebertz-Grün, Ursula. Das andere Mittelalter: Erzählte Geschichte und Geschichtserkenntnis um I300: Studien zu Ottokar von Steiermark, Jans Enikel, Seifried Helbling. Forschungen zur Geschichte der älteren deutschen Literatur 5. Munich, 1984 . 
Loehr, Maja. "Die Grabplatte auf der steirischen Frauenburg und die Ruhestätte Ulrichs von Liechtenstein." MIÖG 65 (I957): 53-69.

Lucas, Henry S. "The Great European Famine of 1315, I3I6 and 1317." Speculum 5 (1930): $34 \mathrm{I}-77$.

Luschin-Ebengreuth, Arnold. "Friesacher Pfennige: Beiträge zu ihrer Münzgeschichte und zur Kenntnis ihrer Gepräge." Numismatische Zeitschrift, n.s., I5 (o.s., 55) (1922): 89-II 8; n.s., I6 (o.s., 56) (1923): 33-144.

Lynch, Joseph H. Godparents and Kinship in Early Medieval Europe. Princeton, N.J., 1986.

Macek, Josef. "Das Turnier im mittelalterlichen Böhmen.” In Fleckenstein, Das ritterliche Turnier, pp. 37I-89.

McFarland, Timothy. "Ulrich von Lichtenstein and the Autobiographical Narrative Form." In Probleme mittelhochdeutscher Erzählformen: Marburger Colloquium 1969, edited by Peter F. Ganz and Werner Schröder, pp. 178-96. Berlin, 1972.

Marx, Erich. "Das Salzburger Vizedomamt Leibnitz." MGSL I 19 ( I979): I-I 42.

Masser, Achim. "Die 'Iwein'-Fresken von Burg Rodenegg in Südtirol und der zeitgenössische Ritterhelm." Zeitschrift für deutsches Altertum und deutsche Literatur I 2 (I983): I77-98.

Mayer, Theodor. "Die Ausbildung der Grundlagen des modernen deutschen Staates im hohen Mittelalter." Historische Zeitschrift I59 (1939): 457-87.

Mertens, Volker. Gregorius Eremita: Eine Lebensform des Adels bei Hartmann von Aue in ihrer Problematik und ihrer Wandlung in der Rezeption. Münchener Texte und Untersuchungen zur deutschen Literatur des Mittelalters 67. Munich, 1978.

— Laudine: Soziale Problematik im "Iwein" Hartmanns von Aue. Beihefte zur Zeitschrift für deutsche Philologie 3. Berlin, 1978.

— . "Das literarische Mäzenatentum der Zähringer." In Die Zähringer: Eine Tradition und ihre Erforschung, edited by Karl Schmid, pp. I17-34. Veröffentlichungen zur ZähringerAusstellung I. Sigmaringen, 1986.

Milnes, Humphrey. "Ulrich von Lichtenstein and the Minnesang." German Life and Letters: A Quarterly Review 17 (1963): 27-43.

Mitterauer, Michael. Karolingische Markgrafen im Südosten: Fränkische Reichsaristokratie und bayerische Stammesadel im österreichischen Raum. AÖG 123. Vienna, 1963.

Moller, Herbert. "The Social Causation of the Courtly Love Complex." Comparative Studies in Society and History I (1958-59): 137-63.

Moraw, Peter. Propyläen Geschichte Deutschlands. Vol. 3. Von offener Verfassung zu gestalteter Verdichtung: Das Reich im späten Mittelalter I25O bis I49O. Berlin, 1985.

Moro, Gotbert. "Zur Geschichte des Lieser- und Katschtales," Carinthia I. 136-38 ( I948): 176-97.

Moÿ, Johannes Graf von. "Die Hintergründe der Fürstungen im Salzburger Domkapitel: Ein Beitrag zur Verfassungsgeschichte des Erzstiftes im I8. Jahrhundert." MGSL I I9 (I979): 23I-59.

Müller, Dominik. "Die Ministerialen des I2. und I3. Jahrhunderts in Salzburg und Gurk." Ph.D. diss., University of Innsbruck, 1904.

9oo Jahre Stift Reichersberg: Augustiner Chorherren zwischen Passau und Salzburg: Ausstellung des Landes Oberösterreich 26. April bis 28. Oktober 1984 im Stift Reichersberg am Inn. Linz, I984.

Novotný, V. "Beiträge zur Geschichte Přemysl Otakars II.” MIÖG 3I (I9I0): 280-30I .

Oberhammer, Eveline. "Untersuchungen zum Konnubium der österreichischen und steirischen Landherren, I 200-I500.” Ph.D. diss., University of Vienna, I973.

Obersteiner, Jakob. Die Bischöfe von Gurk (1072-I822). Aus Forschung und Kunst 5. Klagenfurt, 1969 . 
Oehring, Siglinde. Erzbischof Konrad I. von Mainz im Spiegel seiner Urkunden und Briefe (II6II200). Quellen und Forschungen zur hessischen Geschichte 25. Darmstadt, I 973.

Ogris, Alfred. “Die Anfänge Kärntens.” In Drabek, Österreich im Hochmittelalter, pp. I 29-53.

—. Die Bürgerschaft in den mittelalterlichen Städten Kärntens bis zum Jahre 1335. Das Kärntner Landesarchiv 4. Klagenf urt, 1974.

—_ "Der Kampf des Bistums Gurk um Friesach bis zum Beginn des I4. Jahrhunderts." Carinthia I. I6I/I (197I): I63-74.

Ott, Norbert H., and Wolfgang Walliczek. "Bildprogramm und Textstruktur: Anmerkungen zu den 'Iwein'-Zyklen auf Rodeneck und in Schmalkalden.” In Deutsche Literatur im Mittelalter: Kontakte und Perspektiven: Hugo Kuhn zum Gedenken, edited by Christoph Cormeau, pp. 473-500. Stuttgart, 1979.

Parkin, David. "Kind Bridewealth and Hard Cash: Eventing a Structure.” In Comaroff, Meaning of Marriage Payments, pp. 197-220.

Patze, Hans. "Neue Typen des Geschäftsschriftsgutes im I4. Jahrhundert." In Der deutsche Territorialstaat im I4. Jahrhundert, edited by Hans Patze, I:9-64. 2 vols. VF, I3, I4. Sigmaringen, 1970. Reprint. Sigmaringen, 1986.

Peters, Ursula. "Artusroman und Fürstenhof: Darstellung und Kritik neuerer sozialgeschichtlicher Untersuchungen zu Hartmanns Erec.” Euphorion: Zeitschrift für Literaturgeschichte 69 (1975): $175-96$.

- Frauendienst: Untersuchungen zu Ulrich von Lichtenstein und zum Wirklichkeitsgehalt der Minnedichtung. GAG 46. Göppingen, I97I.

Pferschy, Gerhard. "Zur Beurteilung Siegf rieds von Mahrenberg." In Festschrift Friedrich Hausmann, edited by Herwig Ebner, pp. 367-78. Graz, 1977.

— , ed. Das Werden der Steiermark: Die Zeit der Traungauer. Festschrift zur 80o. Wiederkehr der Erhebung zum Herzogtum. Veröffentlichungen des steiermärkischen Landesarchives Io. Graz, 1980 .

Pickl, Othmar. "Handel und Verkehr in der Steiermark zur Zeit der Traungauer." In Pferschy, Das Werden der Steiermark, pp. 327-54.

Pieper, Michael. Die Funktionen der Kommentierung im "Frauendienst" Ulrichs von Liechtenstein. GAG 35I. Stuttgart, 1982.

Pirchegger, Hans. "Der Besitz des Erzstiftes Salzburg an der Sawe und Enns." ZsHVStm 36 (1943): 59-69.

—_. "Die Herren von Pettau." ZsHVStm 42 ( I95I): 3-36.

- Landesfürst und Adel in Steiermark während des Mittelalters. 3 vols. Forschungen zur Verfassungs- und Verwaltungsgeschichte der Steiermark 12, 13, I6. Graz, I95I-58.

_. Die Untersteiermark in der Geschichte ihrer Herrschaften und Gülten, Städte und Märkte. Buchreihe der südostdeutschen historischen Kommission I0. Munich, 1962.

Posch, Fritz. "Die Anfänge der Steiermark." In Drabek, Österreich im Hochmittelalter, pp. I03-28.

—. "Die Besiedlung und Entstehung des Landes Steiermark." In Pferschy, Das Werden der Steiermark, pp. 23-62.

Probszt, Günther. "Salzburg in der Münz- und Geldgeschichte vergangener Jahrhunderte." MGSL 106 (1966): 27-50.

Puntschart, Paul. "Das 'Inwärts-Eigen' im österreichischen Dienstrecht des Mittelalters: Ein Beitrag zur Eigentums-Theorie." Z(S)RG, GA 43 (1922): 66-IO2.

Rasmo, Nicolò. Pitture murali in Alto Adige. Bolzano, 1973.

Reiffenstein, Ingo. "Rollenspiel und Rollenentlarvung im Frauendienst Ulrichs von Liechtenstein." In Festschrift für Adalbert Schmidt zum 70. Geburtstag, edited by Gerlinde Weiss, pp. 107-20. Stuttgarter Arbeiten zum Germanistik 4. Stuttgart, 1976.

Reindel-Schedl, Helga. "Die Herren von Rohr und ihr Gericht jenseits der Salzach, jenseits der 'Comitie in Tittmaning." " ZsbLG 43 (1980): 329-53. 
“Die Herren von Wispeck." MGSL 122 (1982): 253-86.

. Laufen an der Salzach: Die alt-salzburgischen Pfleggerichte Laufen, Staufeneck, Teisendorf, Tittmoning und Waging. Historischer Atlas von Bayern: Teil Altbayern 55. Munich, I989.

Reuter, Timothy. "Episcopi cum sua militia: The Prelate as Warrior in the Early Staufer Era." In Warriors and Churchmen in the High Middle Ages: Essays Presented to Karl Leyser, edited by Timothy Reuter, pp. 79-94. London, 1992.

— . "Past, Present and No Future in the Twelfth-Century Regnum Teutonicum." In The Perception of the Past in Twelfth-Century Europe, edited by Paul Magdalino, pp. 15-36. London, 1992.

Reynolds, Susan. Kingdoms and Communities in Western Europe, 900-I300. Oxford, 1984.

Rheubottom, D. B. "Dowry and Wedding Celebrations in Yugoslav Macedonia." In Comaroff, Meaning of Marriage Payments, pp. $22 \mathrm{I}-49$.

Richter, Eduard. "Immunität, Landeshoheit und Waldschenkungen.” AÖG 94 (I906): 4I-62.

- Untersuchungen zur historischen Geographie des ehemaligen Hochstifts Salzburg und seiner Nachbargebiete. Innsbruck, 1885.

Riedmann, Josef. “Adelige Sachkultur Tirols in der Zeit von 1290 bis I330." In Adelige Sachkultur des Spätmittelalters, pp. I05-3I.

Rösener, Werner. Peasants in the Middle Ages. Translated by Alexander Stützer. Urbana, Ill., 1992.

Rosenthal, Joel T. Patriarchy and Families of Privilege in Fifteenth-Century England. Philadelphia, I99I.

Rosenwein, Barbara. To Be the Neighbor of Saint Peter: The Social Meaning of Cluny's Property, 909-1049. Ithaca, N.Y., I989.

Roth, Benno. Das älteste Urbar des ehemaligen Augustinerchorherren- und Domstiftes Seckau. Seckauer geschichtliche Studien I. Seckau, I933.

- Besitzgeschichte des ehemaligen Augustinerchorherren- und Domstiftes Seckau, Zeitraum: II4O-I27O. Seckauer geschichtliche Studien 3. Seckau, I933.

Rushing, James A., Jr. "Adventures beyond the Text: Ywain in the Visual Arts." Ph.D. diss., Princeton University, 1988.

Sablonier, Roger. "Zur wirtschaftlichen Situation des Adels im Spätmittelalter." In Adelige Sachkultur des Spätmittelalters, pp. 9-34.

Santifaller, Leo. Das Brixner Domkapitel in seiner persönlichen Zusammensetzung im Mittelalter. Schlern-Schriften: Veröffentlichungen zur Landeskunde von Südtirol 7. Innsbruck, 1924-25.

Scammell, Jean. "The Formation of the English Social Structure: Freedom, Knights, and Gentry, I066-I300." Speculum 68 (I993): 59I-6I8.

Schimmelpfennig, Bernhard. "Zölibat und Lage der 'Priestersöhne' vom I I. bis I4. Jahrhundert." Historische Zeitschrift 227 (1978): I-44.

Schmid, Karl. Gebetsgedenken und adliges Selbstverständnis im Mittelalter: Ausgewählte Beiträge. Festgabe zu seinem sechzigsten Geburtstag. Sigmaringen, 1983.

—_. "Welfisches Selbstverständnis." In Adel und Kirche: Gerd Tellenbach zum 65. Geburtstag dargebracht von Freunden und Schülern, edited by Josef Fleckenstein and Karl Schmid, pp. 389-4I6. Freiburg, 1968. Reprinted in Schmid, Gebetsgedenken, pp. 424-53.

Schmidt, Klaus M. "Späthöfische Gesellschaftsstruktur und die Ideologie des Frauendienstes bei Ulrich von Lichtenstein." Zeitschrift für deutsche Philologie 94 (1975): 37-59.

Schulte, Aloys. Der Adel und die deutsche Kirche im Mittelalter: Studien zur Sozial-, Rechts- und Kirchengeschichte. 2d ed. Stuttgart, 1922. Reprint. Darmstadt, 1958.

Schulz, Knut. "Zum Problem der Zensualität im Hochmittelalter." In Beiträge zur Wirtschaftsund Sozialgeschichte des Mittelalters: Festschrift für Herbert Helbig zum 65. Geburtstag, edited by Knut Schulz, pp. 86-127. Cologne, 1976.

Schupp, Volker. "Kritische Anmerkungen zur Rezeption des deutschen Artusromans anhand 
von Hartmanns 'Iwein': Theorie-Text-Bildmaterial." Frühmittelalterliche Studien: Jahrbuch des Instituts für Frühmittelalterforschung der Universität Münster 9 ( I 975): 405-42.

Sonnleitner, Käthe: "Die Stellung der Kinder von Unfreien im Mittelalter in Salzburg, Steiermark und Kärnten." MGSL I23 (1983): I 49-66.

Sparber, Anselm. "Aus dem Leben und Wirken des Brixner Fürstbischofs Konrad von Rodank (1200-1216)." Der Schlern 34 (1960): 238-45.

_. Die Brixner Fürstbischöfe im Mittelalter: Ihr Leben und Wirken. Bolzano, 1968.

Spechtler, Franz Viktor. "Probleme um Ulrich von Liechtenstein: Bemerkungen zu historischen Grundlagen, Untersuchungsaspekten und Deutungsversuchen.” In Ebenbauer, Knapp, and Strasser, Österreichische Literatur zur Zeit der Babenberger, pp. 218-32.

_ . "Untersuchungen zu Ulrich von Liechtenstein." Habilitationsschrift, University of Salzburg, 1974.

Spindler, Max, ed. Handbuch der bayerischen Geschichte. Vol. I. Das alte Bayern: Das Stammesherzogtum bis zum Ausgang des I2. Jahrhunderts. Vol. 2. Das alte Bayern: Der Territorialstaat vom Ausgang des I2. Jahrhunderts bis zum Ausgang des I8. Jahrhunderts. Munich, 1967-69.

Spreitzhofer, Karl. Georgenberger Handfeste: Entstehung und Folgen der ersten Verfassungsurkunde der Steiermark. Steiermärkisches Landesarchiv, n.s., 3. Graz, 1986.

Störmer, Wilhelm. "Adel und Ministerialität im Spiegel der bayerischen Namengebung (bis zum I3. Jahrhundert): Ein Beitrag zum Selbstverständnis der Führungsschichten.” Deutsches Archiv für Erforschung des Mittelalters 33 (1977): 84-152.

- Früher Adel: Studien zur politischen Führungsschicht im fränkisch-deutschen Reich vom 8. bis II. Jahrhundert. Monographien zur Geschichte des Mittelalters 6. Stuttgart, I 973.

— . "Frühmittelalterliche Grundherrschaft bayerischer Kirchen (8.-IO. Jahrhundert)." In Strukturen der Grundherrschaft im frühen Mittelalter, edited by Werner Rösener, pp. 370-4IO. VMPIG 92. Göttingen, I989.

— . "König Artus als aristokratisches Leitbild während des späteren Mittelalters, gezeigt am Beispielen der Ministerialität und des Patriziats." ZsbLG 35 (1972): 946-7I.

Stolz, Otto. "Das Wesen der Grafschaft im Raume Oberbayern-Tirol-Salzburg." ZsbLG I5 (I949): 68-I09.

Stuard, Susan Mosher. A State of Deference: Ragusa/Dubrovnik in the Medieval Centuries. Philadelphia, 1992.

Thomas, Heinz. "Julius Caesar und die Deutschen: Zu Ursprung und Gehalt eines deutschen Geschichtsbewusstseins in der Zeit Gregors VII. und Heinrichs IV.” In Die Salier und das Reich, edited by Stefan Weinfurter, 3 vols. 3:245-77. Sigmaringen, I99I .

Thum, Bernd. "Ulrich von Lichtenstein: Höfische Ethik und soziale Wirklichkeit." Ph.D. diss., University of Heidelberg, 1968.

Töchterle, Georg. "Die Herren von Rodank und Schöneck." Der Schlern I2 (I93I): I8-29, 93-I00, I4I-45.

Trautz, Fritz. "Noblesse allemande et noblesse anglaise: Quelques points de comparaison." In Famille et parenté dans l'Occident médiéval: Actes du Colloque de Paris (6-8 juin 1974) organisé par l'Ecole pratique des hautes études (VIe section) en collaboration avec le Collège de France et l'Ecole française de Rome, edited by Georges Duby and Jacques Le Goff, pp. 63-8I . Collection de l'Ecole française de Rome 30. Rome, 1977.

Tropper, Christine. "Die Stifter des Hemma-Freskos in Zweinitz: Zum Problem Gurktaler Adel und Gurker Domkapitel in der I. Hälfte des I5. Jahrhunderts." Carinthia I. I80 (I990): 285-301.

Tyroller, Franz. Genealogie des altbayerischen Adels im Hochmittelalter. Genealogische Tafeln zur mitteleuropäischen Geschichte 4. Göttingen, 1962.

_ . "Die Grafschaften des Isengaues." Oberbayerisches Archiv für vaterländische Geschichte 80 ( I955): 45-I02. 
Voit, Gustav. Der Adel am Obermain: Genealogie edler und ministerialer Geschlechter vom II. bis I4. Jahrhundert. Die Plassenburg: Schriften für Heimatforschung und Kulturpflege in Ostf ranken 28. Kulmbach, 1969.

Volk, Otto. Salzproduktion und Salzhandel mittelalterlicher Zisterzienserklöster. VF, Sonderband 30. Sigmaringen, 1984 .

Wagner, Hans, and Hebert Klein. "Salzburgs Domherren von I30o bis I 5I4." MGSL 92 ( 1952 ): I-8I.

Wanderwitz, Heinrich. "Traditionsbücher bayerischer Klöster und Stifte." Archiv für Diplomatik: Schriftsgeschichte, Siegel- und Wappenkunde 24 (1978): 359-80.

Wapnewski, Peter. Hartmann von Aue. 4th ed. Sammlung Metzler 17. Stuttgart, 1969.

Weinfurter, Stefan, ed. Die Salier und das Reich. 3 vols. Sigmaringen, I99I.

—. Salzburger Bistumsreform und Bischofspolitik im I2. Jahrhundert: Der Erzbischof Konrad I. von Salzburg (IIO6-II47) und die Regularkanoniker. Kölner historische Abhandlungen 24. Cologne, I975.

Wendehorst, Alfred. "Bischöfe und Bischofskirchen von Würzburg, Eichstätt und Bamberg." In Weinfurter, Die Salier und das Reich, 2:225-50.

Werner, Karl Ferdinand. "Heeresorganisation und Kriegführung im deutschen Königreich des Io. und I I . Jahrhunderts." In Structures politiques du monde franc (VIe-XIIe siècles): Etudes sur les origines de la France et de l'Allemagne, pp. 79I-843. London, 1979.

White, Stephen D. Custom, Kinship, and Gifts to Saints: The "Laudatio Parentum" in Western France, IO5O-II5O. Chapel Hill, N.C., I988.

Widmann, Hans. Geschichte Salzburgs. 3 vols. Deutsche Landesgeschichten 9. Gotha, 1907-14.

Zallinger, Otto von. Ministeriales und Milites: Untersuchungen ueber die ritterlichen Unfreien zunaechst in baierischen Rechtsquellen des XII. und XIII. Jahrhunderts. Innsbruck, I878.

Zillner, Franz Valentin. "Salzburgische Geschlechterstudien: II. Izling-Fischach-BergheimRadeck.” MGSL 19 (I879): I-65.

— . "Salzburgische Geschlechterstudien: III. Die Werfener Burggrafen." MGSL 2I ( I88I): 24-79.

Zotz, Thomas. "Die Formierung der Ministerialität." In Weinfurter, Die Salier und das Reich, 3:3-50.

- "Milites Christi: Ministerialität als Träger der Kanonikerreform." Quellen und Abhandlungen zur mittelrheinischen Kirchengeschichte 68 (1992): 30I-28. 
